\section{Semiconductor nanocrystal conjugates, FISH and pH}

To the editor: Properties of high luminescence, reduced photobleaching and single-wavelength excitation make quantum dots (QDs) ideal for bioimaging ${ }^{1}$. Fluorescence in situ hybridization (FISH) applications have been limited for lack of reagents, stable surface treatments ${ }^{2}$ and optimization. We previously demonstrated QD-FISH detection of DNA probes with (CdSe)/ZnSstreptavidin conjugates ${ }^{3}$. Here we show $\mathrm{pH}$ effects on fluorescence of QD-detected hybridization signals in FISH experiments that are not evident in solution experiments.

FISH (Fig. 1a) revealed major hybridization sites irrespective of the type of conjugate (organic or nanocrystal). We were able to detect the centromeres of other human chromosomes with organic fluorophores, but not with quantum dots (Fig. 1a).

We investigated the effects of $\mathrm{pH}$ on fluorescence with biotinylated total human genomic DNA probes in FISH experiments. At each $\mathrm{pH}$, cell manipulations were identical before the final incu-

a

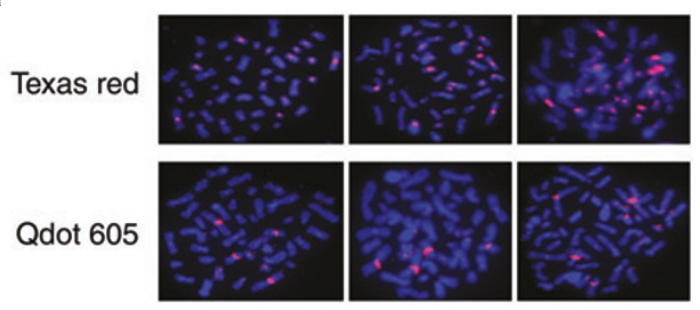

b

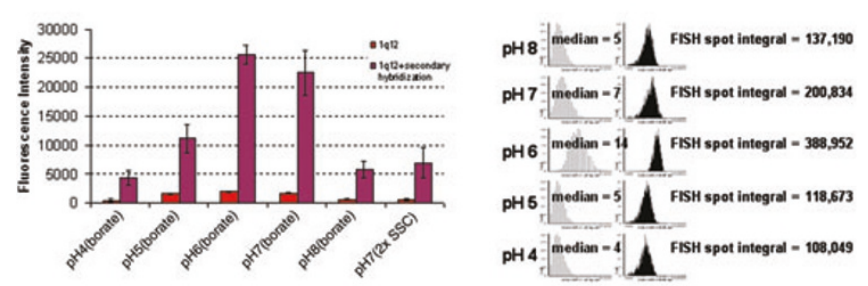

Figure 1 | pH dependence of fluorescence intensity in FISH experiments with total genomic DNA detected by Texas red and QDs. (a) Distribution of FISH signals detected with Texas red-streptavidin conjugate (top); and distribution of Qdot 605-streptavidin detected FISH signals (bottom). I mages from three different experiments are shown to demonstrate the consistency of the labeling difference. Bar, $10 \mu \mathrm{m}$. (b) Signal intensity digital quantitation of FISH signal at chromosome band 1 q12 or total signal ( $n=3$ cells, \pm s.d.). In $\mathbf{a}$ and $\mathbf{b}$, fluorescence intensity was measured by digital imaging with mercury lamp illumination. (c) Identical FISH experiment quantitated by LSC. Left, total number of spots in each cell; right, intensity of total FISH signal. ( $n=5,000-10,000$ cells with median value, left or integral, right). bation with fluorophore-streptavidin conjugates. Fluorescence intensities of chromosome band 1q12 (ref. 4) and total chromosomal signal were plotted (Fig. 1b). QD fluorophore intensities varied with $\mathrm{pH}$ in two different imaging systems (Fig. 1b,c). QD signals were optimal at $\sim \mathrm{pH} 6-7$, and diminished at $\mathrm{pH} 4$ and pH 8 (Fig. 1b, manually quantified FISH). Standard deviations at pH 6 and pH 7 overlapped (Fig. 1b). Laser scanning cytometry (LSC) experiments confirmed this finding (5,000-10,000 cells at each pH; Fig. 1c). LSC measurements included the number of signals in each cell and the integrated density of all QD signals (Fig. 1c). These data confirmed that borate buffers at $\mathrm{pH} 6$ and 7 gave superior fluorescence intensity. Quantification showed fluorescence intensity was greater at $\mathrm{pH} 6$ than $\mathrm{pH} 7$ by twofold (Fig. 1c). Previously, we ${ }^{3}$ noted qualitative distribution differences of fluorescence sites between fluorescein isothiocyanate (FITC) and QD detection systems. Here we quantitatively show that spot number at $\mathrm{pH} 6$ was greater than at lower or higher pH (Fig. 1c). Rescanning FISH/LSC experiments demonstrated that QD fluorescence was more stable ( $98 \%$ after five scans) than Alexa Fluor 488 (75\% after five scans), extending this analysis to high-throughput imaging (Supplementary Fig. 1 online). Singlecell FISH with dynamic changes in $\mathrm{pH}$ via microfluidics using a third imaging system also confirmed this (Supplementary Data and Supplementary Fig. 2 online). Thus, fluorescence intensity of streptavidin-QD conjugates used to detect FISH hybridization probes varied with $\mathrm{pH}$ of the final incubation buffer.

Control solution experiments with QD conjugates in the presence or absence of DNA showed no $\mathrm{pH}$ dependence (Supplementary Data). It is unclear why fluorescence intensity of the QD-streptavidin probe in the FISH format exhibited $\mathrm{pH}$ dependence. $\mathrm{pH}$ control, however, may allow optimization of QD fluorophores in clinically useful formats such as FISH and other hybridization-based assays.

Additional information is available in Supplementary Methods online.

Note: Supplementary information is available on the Nature Methods website.

\section{Yan Xiao ${ }^{1}$, William G Telford ${ }^{2}$, J Christopher Ball ${ }^{3}$, Laurie E Locascio ${ }^{3} \&$ Peter E Barker ${ }^{1}$}

${ }^{1}$ DNA Technologies Group, Biotechnology Division, National Institute of Standards and Technology, Gaithersburg, Maryland 20899, USA. ${ }^{2}$ Experimental Transplantation and Immunology Branch, Center for Cancer Research, National Cancer Institute, National Institutes of Health, Bethesda, Maryland 20892, USA. ${ }^{3}$ Molecular Spectrometry and Microfluidic Methods Group, Analytical Chemistry Division, National Institute of Standards and Technology, Gaithersburg, Maryland 20899, USA.

e-mail: peter.barker@nist.gov

1. Alivisatos, P. Nat. Biotechnol. 22, 47-52 (2004).

2. Pathak, S., Choi, S-K., Arnheim, N. \& Thompson, M.E. J. Am. Chem. Soc. 123, 4103-4104 (2001)

3. Xiao, Y. \& Barker, P.E. Nucleic Acids Res. 32, e28 (2004). 